\title{
66 Leistungsstoffwechsel und Insulinwirkung
}

\author{
(c) Springer-Verlag GmbH Deutschland, ein Teil von Springer Nature 2018 \\ D. Mathias, Fit und gesund von 1 bis Hundert \\ https://doi.org/10.1007/978-3-662-56307-6_66
}

Während die Adrenalinausschüttungen belastungsabhängig verschieden hoch ausfallen, bleiben die Insulinspiegel beim Sport zunächst im Normalbereich und sinken bei länger andauernden Aktivitäten ab. Da jedoch leistungsbedingt der Glucosedurchsatz erheblich angehoben ist, kommen trainierte Menschen für den Verbrauch einer bestimmten Glucosemenge offenbar mit weniger Insulin aus. Das liegt einmal daran, dass durch regelmäßige körperliche Anstrengungen Insulin wegen einer dann verbesserten Ansprechrate der in den Muskelzellen lokalisierten Insulinrezeptoren eine höhere Effektivität besitzt. Und zum anderen werden unter Belastung für die Einschleusung von Glucose in die Zellen hormonunabhängige Transportmechanismen begünstigt.

Die Verwertung der intramuskulären Glucosevorräte kann dann schon durch die Muskelkontraktionen selbst erfolgen.

Nur die Mobilisierung der extramuskulären Glucosedepots zugunsten der Muskulatur unterliegt unter diesen Bedingungen noch ganz der Kontrolle des Insulins (s. aber $>$ Kap. 64).

Der Umstand, dass es bei sportlichen Leistungen eher zu verminderten Insulinausschüttungen kommt, ist auf den ersten Blick ein erstaunliches Phänomen. Bei näherem Hinsehen entpuppt sich dies aber wieder als ein sinnvoller Anpassungsmechanismus der Natur, hier an den Leistungsstoffwechsel. Denn würden während körperlicher Tätigkeiten die Insulinsekretionen hoch sein, könnte die notwendige Eigensynthese von Glucose in der Leber nicht in Gang kommen und die Belastung müsste frühzeitig abgebrochen werden. Käme dann noch der allein schon durch den Belastungsreiz ausgelöste, insulinunabhängige Einstrom von Glucose in die Muskelzelle hinzu, entstünde eine dauerhafte Gefahr der Unterzuckerung mit schwerwiegenden gesundheitlichen Beeinträchtigungen.

Durch die bei sportlichen Anstrengungen erniedrigten Insulinspiegel werden Reinigung und Erneuerung der Zellen gefördert. 